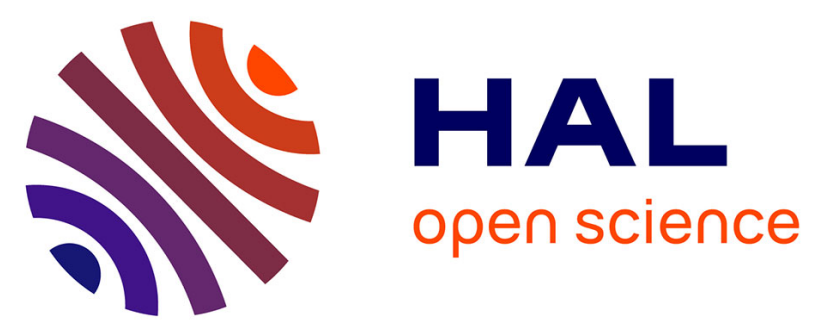

\title{
Homogenized electromechanical coefficients and effective parameters of 1-3 piezocomposites for ultrasound imaging transducers
}

Pierre Tize Mha, Pierre Maréchal, Guy Edgar Ntamack, Saâd Charif d'Ouazzane

\section{To cite this version:}

Pierre Tize Mha, Pierre Maréchal, Guy Edgar Ntamack, Saâd Charif d'Ouazzane. Homogenized electromechanical coefficients and effective parameters of 1-3 piezocomposites for ultrasound imaging transducers. Physics Letters A, 2021, 408, pp.127492. 10.1016/j.physleta.2021.127492 . hal$03263184 \mathrm{v} 2$

\section{HAL Id: hal-03263184 \\ https://hal.science/hal-03263184v2}

Submitted on 21 Jul 2021

HAL is a multi-disciplinary open access archive for the deposit and dissemination of scientific research documents, whether they are published or not. The documents may come from teaching and research institutions in France or abroad, or from public or private research centers.
L'archive ouverte pluridisciplinaire HAL, est destinée au dépôt et à la diffusion de documents scientifiques de niveau recherche, publiés ou non, émanant des établissements d'enseignement et de recherche français ou étrangers, des laboratoires publics ou privés. 


\title{
Homogenized electromechanical coefficients and effective parameters of 1-3 piezocomposites for ultrasound imaging transducers
}

\author{
P. Tizé Mha ${ }^{1}$, P. Maréchal ${ }^{2}$, G.E. Ntamack ${ }^{1}$, S. Charif d'Ouazzane ${ }^{3}$
}

\begin{abstract}
Analytical models can be useful tools to develop efficient transducers dedicated to a specific application field. This work proposes a homogenization technique for establishing the effective coefficients and parameters of 1-3 piezocomposite whose both phases are piezoelectrically active. Such piezocomposite materials may have homogenized electromechanical properties resulting from positive hybrid effect. A numerical simulation of the effective parameters resulting from a PZT-5A / PVDF-TrFE composition shows that, volume fraction influences significantly on the properties of the piezocomposite and consequently on its behavior in service. As an end-user application for ultrasound imaging, those piezocomposite materials are effectively integrated in a piezocomposite transducer, coupled with a dedicated backing. The resulting characteristics in terms of impulse response and associated electroacoustic echo are compared and discussed for typical configurations. A method for the optimization of the design of such piezocomposite transducer is presented. Specific estimators based on the bandwidth flatness $B W F$ and bandwidth amplitude product $B W A$ are proposed as stable and smooth criteria for an optimization procedure.
\end{abstract}

Keywords: Homogenization, connectivity, electromechanical property, piezoelectric material

\section{Introduction}

Piezoelectricity is a physical property of active materials with various applications as transducers, vibration control and positioning actuator applications [1, 2]. By promoting the flow of charges in a piezoelectric material bonded to a structure, it is possible to extract small powers (going to microwatt or milliwatt). In general, their use tends to develop in mobile or embedded electronic devices. The commissioning of a piezoelectric material requires a study and analysis of its properties beforehand. Indeed, previous studies have shown that piezoelectric materials consisting of several phases (active and passive) with particular characteristics do not resist long loading in service. With the advance of technology, man is constantly searching for materials that can play an important role in modernising the designs of intelligent structures and systems. Among the materials exploited so far, it appears that the active materials have shown their proof. Among the classes of active materials, piezoelectric materials are widely used in several fields of application and specifically for the control of vibrating structures such as acoustic transducers and the recovery of energy [3, 4]. The electromechanical coupling combined with the high performance of monolithic materials has allowed them to be clas-

\footnotetext{
Email addresses: tizemha@yahoo.com (P. Tizé Mha), pierre.marechal@univ-lehavre.fr (P. Maréchal)

${ }^{1}$ GMMA: Groupe de Mécanique, Matériaux et Acoustique, Département de Physique, Faculté des Sciences, Université de Ngaoundéré B.P. 454 Ngaoundéré, Cameroun.

${ }^{2}$ LOMC: Laboratoire Ondes et Milieux Complexes, UMR 6294 CNRS, Université du Havre, France.

${ }^{3}$ LMTM: Laboratoire de Mécanique, Thermique et Matériaux, École Nationale Supérieure des Mines, Rabat, ENSM, B.P. 753 Rabat, Maroc.
}

sified as sensors and actuators. However, these materials are fragile and therefore may exhibit cracking at the least amount of stress. To overcome this problem, the concept of piezocomposite is put in place to replace a piezoelectric material all alone like monolithic ceramics. In this case a combination is made between the fibres and a ductile matrix such as polymer [5]. Some advantages are remarkable in a piezocomposite which is not found in a monolithic. These advantages include high sensitivity, a wide range of mechanical properties, very low density, low impedance, high strength [2, 6]. It is therefore clear that piezocomposites provide good answers in terms of applications as in medical transducers, in submarines as hydrophones used to detect acoustic waves and many other applications [7, 8, 9]. A wide range of possible applications have been investigated in energy harvesting [10, 11], tunable filtering for radio frequency telecommunications [12, 13], high power transducers [14]. Several models exist in the literature for the prediction of the electromechanical behaviour of piezocomposites. These models can be divided into two groups: analytical and numerical models. In the group of analytical models we can cite the model of Newnham et al. [15] developed on the basis of the notions of serial/parallel connectivity for pyroelectric piezocomposites. Banno [16] formulates an analytical model to study the coupling of closed and open pore ceramics based on the modified cube model. In order to better understand the reality described by the experiments referring to the works mentioned above under different volumetric fractions, please consult the works of Furukawa et al. [17], Steinhausen et al. [18], Chan et al [19, 20]. The model of Dunn and Taya [21] makes it possible to predict the behaviour of piezocomposite by evaluating its electromechanical properties using Mori-Tanaka's microdiffer- 
ential theories and the principle of dilute micromechanics. The Kuo and Huang [22] method based on the notion of anisotropy of $3 \mathrm{D}$ inclusions evaluates the properties of these inclusions as the properties related to the phases, the orientation of the angles, the volume fraction and the shape of the inclusions. As for Nan et al. [23] they proposed a technique for calculating the coefficients of 1-3 piezocomposite in which the piezoelectric phase $\mathrm{PZT}\left(\mathrm{Pb}(\mathrm{Zr} / \mathrm{Ti}) \mathrm{O}_{3}\right.$ composite) lead rod or zirconate fibres (PZT/epoxy) are randomly arranged in a matrix using the Green method. Odegard [24] expands the TM fomulation for the prediction of the mechanical properties of certain composites. Kar-Gupta and Venkatesh [25] develop a model that takes into account the anisotropy of the fibres always to study the behaviour of piezocomposite where both phases are active. As highlighted by Chan, Ng and Choy [19, 20] in the case of two-active-phase composites, the resulting piezoelectric coefficients can be enhanced if the phases exhibit opposite polarization. Such a configuration would have a considerable impact on the effective piezoelectric effects, rising the interest of a study under such circumstances. The homogenized coefficients of a composite in general are related to the structure of its constituent cell; in other word, to the properties of the elements of that cell. [3, 26, 27, 28, 29]. Among the multi-scale methods, the homogenization method leads to macroscopic characteristics of a heterogeneous material from those of elementary homogeneous phases. In this work, a homogenization method is used for the determination of the effective electromechanical coefficients. It is an extension of the Smith model [3] to the case of a two piezoelectric phases. The main paragraphs of this work are as follows: Section 2 presents the connectivities types where the Smith model is generalized. Results and discussion are presented in Section 3, and followed by underwater application in Section 4. An end-user case of study is developed and discussed in Section 5, where the generalized Smith model is used as an input of the KLM (for Krimholtz, Leedom \& Mattei)) model and applied to piezocomposite transducers for imaging.

\section{Connectivity}

To describe how the phases are arranged in a piezocomposite [15] introduced the notion of connectivity. Suppose a cube isolated from a two-phase piezocomposite. The number of directions by which this cube can be crossed from one side to another following the line of a stop without leaving a considered phase represents a connectivity. The presented connectivities are illustrating the 16 possible combinations of connectivities in 3 dimensions, i.e. $(\alpha, \beta) \in\{0,1,2,3\}$ for a two-phase composite material, with $(\alpha, \beta)$ the connectivities of phase 1 and 2 respectively. Since the symmetric cases $(\alpha, \beta)$ are resulting from a permutation of phases 1 and 2 , the 16 possibilities are reduced to 10 illustrations only. The different connectivities are illustrated in the Figure 1 below, with the shaded phase 1 and the clear phase 2 .

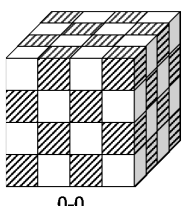

$0-0$

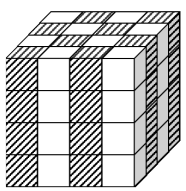

$1-1$

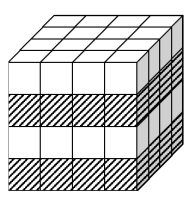

2-2
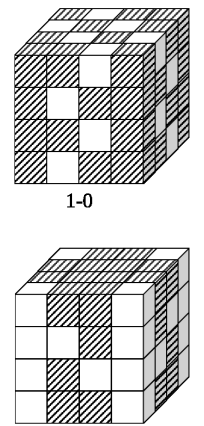

2-1

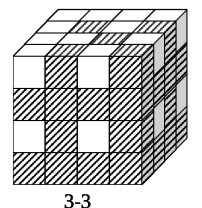

3-3

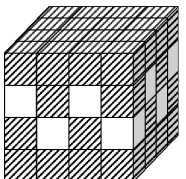

2-0

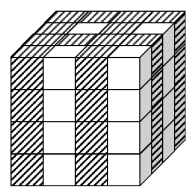

3-1

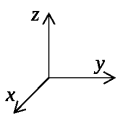

Figure 1: Connectivities of two-phases composite: phase 1 (shaded) and phase 2 (clear), from Newnham [15]

\subsection{Generalized Smith model}

To establish his model, Smith [1] considers a thick 1-3 piezocomposite and chooses to work in plane stress and plane strain. This piezocomposite is composed by two phases: the passive matrix and the active fiber. As a result, this model gives the effective properties of the piezocomposite. Piezoelectricity makes it possible to establish a relationship between the mechanical and electrical properties of a piezo-composite and, as an example, the mechanical deformation and the electric field $[S, E]^{t}$, are linked to the mechanical stress and the electrical displacement $[T, D]^{t}$ by the following matrix relationship:

$$
\left[\begin{array}{l}
T \\
D
\end{array}\right]=\left[\begin{array}{cc}
{\left[c^{E}\right]} & -[e]^{t} \\
{[e]} & {\left[\varepsilon^{S}\right]}
\end{array}\right]\left[\begin{array}{l}
S \\
E
\end{array}\right] \equiv[T D]=[K][S E]
$$

This writing of the generalized stiffness matrix $[K]$ will further be denoted with a superscript $\{m, f\}$ for the matrix and filler phases respectively. The effective piezocomposite properties will be overlined, and the generalized stiffness matrix will be denoted as $[K]$. As part of our study, we consider moreover the $z$ axis as the polarization axis, then the generalized stiffness matrix $[K]$ in detailed form for active phase, is as follows:

$\left[\begin{array}{l}T_{1} \\ T_{2} \\ T_{3} \\ T_{4} \\ T_{5} \\ T_{6} \\ D_{1} \\ D_{2} \\ D_{3}\end{array}\right]=\left[\begin{array}{ccccccccc}c_{11}^{E} & c_{12}^{E} & c_{13}^{E} & 0 & 0 & 0 & 0 & 0 & -e_{31} \\ c_{12}^{E} & c_{11}^{E} & c_{13}^{E} & 0 & 0 & 0 & 0 & 0 & -e_{31} \\ c_{13}^{E} & c_{13}^{E} & c_{33}^{E} & 0 & 0 & 0 & 0 & 0 & -e_{33} \\ 0 & 0 & 0 & c_{44}^{E} & 0 & 0 & 0 & -e_{15} & 0 \\ 0 & 0 & 0 & 0 & c_{44}^{E} & 0 & -e_{15} & 0 & 0 \\ 0 & 0 & 0 & 0 & 0 & c_{66}^{E} & 0 & 0 & 0 \\ 0 & 0 & 0 & 0 & e_{15} & 0 & \varepsilon_{11}^{S} & 0 & 0 \\ 0 & 0 & 0 & e_{15} & 0 & 0 & 0 & \varepsilon_{11}^{S} & 0 \\ e_{31} & e_{31} & e_{33} & 0 & 0 & 0 & 0 & 0 & \varepsilon_{33}^{S}\end{array}\right]\left[\begin{array}{l}S_{1} \\ S_{2} \\ S_{3} \\ S_{4} \\ S_{5} \\ S_{6} \\ E_{1} \\ E_{2} \\ E_{3}\end{array}\right]$

where $[T D]=[T, D]^{t}$ is the generalized stress vector including $T$ and $D$, the stress components and the electrical dis- 
placement components respectively; $[S E]=[S, E]^{t}$ is the generalized strain vector including $S$ and $E$, the strain components and the electrical field components, respectively; the generalized stiffness matrix $[K]$ including $\left\{c^{E}, e, \varepsilon^{S}\right\}$ with $c^{E}$ the stiffness components at constant electrical field, $e$ the piezoelectric components, and $\varepsilon^{S}$ the dielectric components at constant strain. The considered piezoelectric materials are in the $4 \mathrm{~mm}$ symmetry class. Thus, their generalized stiffness matrix is with symmetric and having 25 coefficients, and among them 11 independent coefficients. One can notice that for the case of the passive phase (matrix), there is no piezoelectric effect, i.e $e_{i j}=0$ . That is the restriction of the Smith model, where one of the two constitutive phases is passive. This limitation will be overloaded in this study, where both phases will be considered as possibly active phases.

\subsubsection{Assumptions}

An analysis with these nine equations for both phases seems complex and requires simplification. Thus, six approximations are considered:

\section{- First approximation:}

Mechanical deformation and electrical field are considered independent of $x$ and $y$ in each phase. This approximation makes it possible to simplify the relations in both phases, denoted with the $\{m, f\}$ exponents, for the two phases: matrix (phase 1) and fiber (phase 2), respectively.

\section{- Second approximation:}

It is supposed that the strain along the $x$ direction is the same as that along the $y$ direction, both in the phases 1 and 2 so that $S_{1}^{m}=S_{2}^{m}$ and $S_{1}^{f}=S_{2}^{f}$. Moreover, the effective strain $S_{1}$ and $S_{2}$, along the $x$ and $y$ directions respectively is resulting from a volume averaged sum of those of the phases, so that $S_{1}=S_{2}=v_{m} S_{1}^{m}+v_{f} S_{1}^{f}$. In addition, the effective strain along the $x$ and $y$ directions is neglected, so that $S_{1}=S_{2}=0$. Similarly, the effective electric field along the $x$ and $y$ directions is neglected, so that $E_{1}=E_{2}=0$. Those assumptions are leading to the equalities $T_{1}=T_{2}$, and $D_{1}=D_{2}$, and resulting in the following simplified expressions for the two constitutive phases:

$$
\left\{\begin{array}{l}
T_{1}^{\{m, f\}}=\left(c_{11}^{\{m, f\}}+c_{12}^{\{m, f\}}\right) S_{1}^{\{m, f\}}+c_{13}^{\{m, f\}} S_{3}^{\{m, f\}}-e_{31}^{\{m, f\}} E_{3}^{\{m, f\}} \\
T_{3}^{\{m, f\}}=2 c_{13}^{\{m, f\}} S_{1}^{\{m, f\}}+c_{33}^{\{m, f\}} S_{3}^{\{m, f\}}-e_{33}^{\{m, f\}} E_{3}^{\{m, f\}} \\
D_{3}^{\{m, f\}}=2 e_{31}^{\{m, f\}} S_{1}^{\{m, f\}}+e_{33}^{\{m, f\}} S_{3}^{\{m, f\}}+\varepsilon_{33}^{\{m, f\}} E_{3}^{\{m, f\}}
\end{array}\right.
$$

\section{- Third approximation:}

The two phases move together along the $z$ axis:

$$
S_{3}^{m}=S_{3}^{f}=S_{3}
$$

\section{- Four approximation:}

The electric field is considered identical in both phases along the $\mathrm{z}$ axis.

$$
E_{3}^{m}=E_{3}^{f}=E_{3}
$$

\section{- Fifth approximation:}

The lateral interactions between phases are developed along the $x$ axis:

$$
S_{1}=v_{m} S_{1}^{m}+v_{f} S_{1}^{f}=0
$$

where $v_{f}=1-v_{m}$ is the volume fraction of piezoelectric phase. Moreover, the stress along the $x$ axis is considered identical in both phases:

$$
T_{1}^{m}=T_{1}^{f}=T_{1}
$$

On the one hand, from equations (3), 4), (5), (6) and (7), we get:

$$
\left\{\begin{array}{l}
S_{1}^{f}=v_{m} \frac{\left(c_{13}^{m}-c_{13}^{f}\right) S_{3}+\left(e_{31}^{f}-e_{31}^{m}\right) E_{3}}{v_{f}\left(c_{11}^{m}+c_{12}^{m}\right)+v_{m}\left(c_{11}^{f}+c_{12}^{f}\right)} \\
S_{1}^{m}=-\frac{v_{f}}{v_{m}} S_{1}^{f}
\end{array}\right.
$$

On the other hand, from equations $(3),(4),(5),(6)$ and (8), we get:

$$
T_{1}^{f}=v_{m} \frac{c_{11}^{f}+c_{12}^{f}}{c_{m f}}\left(\left(c_{13}^{m}-c_{13}^{f}\right) S_{3}+\left(e_{31}^{f}-e_{31}^{m}\right) E_{3}\right)+c_{13}^{f} S_{3}-e_{31}^{f} E_{3}
$$

with:

$$
c_{m f}=v_{f}\left(c_{11}^{m}+c_{12}^{m}\right)+v_{m}\left(c_{11}^{f}+c_{12}^{f}\right)
$$

The identification of the effective properties from $T_{1}^{f}=$ $\bar{c}_{13}^{E} S_{3}-\bar{e}_{31} E_{3}$ results in:

$$
\left\{\begin{array}{l}
\bar{c}_{13}^{E}=c_{13}^{f}\left(v_{f} \frac{c_{11}^{m}+c_{12}^{m}}{c_{m f}}+v_{m} \frac{\left(c_{11}^{f}+c_{12}^{f}\right)}{c_{m f}} \frac{c_{13}^{m}}{c_{13}^{f}}\right. \\
\bar{e}_{31}=e_{31}^{f}\left(v_{f} \frac{c_{11}^{m}+c_{12}^{m}}{c_{m f}}+v_{m} \frac{\left(c_{11}^{f}+c_{12}^{f}\right)}{c_{m f}} \frac{e_{31}^{m}}{e_{31}^{f}}\right.
\end{array}\right)
$$

\section{- Sixth approximation:}

The axial interactions between phases are developed along the $z$ axis:

$$
\left\{\begin{array}{l}
T_{3}=v_{f} T_{3}^{f}+v_{m} T_{3}^{m} \\
D_{3}=v_{f} D_{3}^{f}+v_{m} D_{3}^{m}
\end{array}\right.
$$

On other hand, from equations (3) and (8) we have also:

$$
\left\{\begin{array}{l}
T_{3}^{f}=\left(c_{33}^{f}+2 v_{m} \frac{c_{13}^{f}\left(c_{13}^{m}-c_{13}^{m}\right)}{c_{m f}}\right) S_{3}-\left(e_{33}^{f}-2 v_{m} \frac{c_{13}^{f}\left(e_{31}^{f}-e_{31}^{m}\right)}{c_{m f}}\right) E_{3} \\
D_{3}^{f}=\left(e_{33}^{f}+2 v_{m} \frac{e_{31}^{f}\left(c_{13}^{m}-c_{13}^{m}\right)}{c_{m f}}\right) S_{3}+\left(\varepsilon_{33}^{f}+2 v_{m} \frac{e_{31}^{f}\left(e_{31}^{f}-e_{31}^{m}\right)}{c_{m f}}\right) E_{3}
\end{array}\right.
$$

and reciprocally for $\left\{T_{3}^{m}, D_{3}^{m}\right\}$ by switching the $\{m, f\}$ exponents in equation (13). 


\subsubsection{Effective properties}

In the literature, from several applications, those effective electroacoustic properties have made it possible to evaluate the performance of piezocomposites [3, 30, 31, 32]. The typically interest for end-users developers are the actual parameters including effective piezoelectric electromechanical coupling coefficient $\bar{k}_{t}$, the acoustic impedance $\bar{Z}_{L}$ and the stiffened longitudinal velocity $\bar{c}_{L}$. Thus, we obtain the effective coefficients which define the Smith model according to the relations hereafter:

$$
\left\{\begin{array}{l}
T_{3}=\bar{c}_{33}^{E} S_{3}-\bar{e}_{33} E_{3} \\
D_{3}=\bar{e}_{33} S_{3}+\bar{\varepsilon}_{33}^{S} E_{3}
\end{array}\right.
$$

where:

$$
\left\{\begin{array}{l}
\bar{c}_{33}^{E}=\left(v_{f} c_{33}^{f}+v_{m} c_{33}^{m}\right)-2 \frac{v_{f} v_{m}}{c_{m f}}\left(c_{13}^{f}-c_{13}^{m}\right)^{2} \\
\bar{e}_{33}=\left(v_{f} e_{33}^{f}+v_{m} e_{33}^{m}\right)-2 \frac{v_{f} v_{m}}{c_{m f}}\left(e_{31}^{f}-e_{31}^{m}\right)\left(c_{13}^{f}-c_{13}^{m}\right) \\
\bar{\varepsilon}_{33}^{S}=\left(v_{f} \varepsilon_{33}^{f}+v_{m} \varepsilon_{33}^{m}\right)+2 \frac{v_{f} v_{m}}{c_{m f}}\left(e_{31}^{f}-e_{31}^{m}\right)^{2}
\end{array}\right.
$$

As a remark, the opposite signs of the $e_{31}$ piezoelectric coefficients in the two constitutive phases can lead to a more significant transverse contribution since its contribution is involved in the effective piezoelectric coefficient $e_{33}$ and in the effective dielectric coefficient $\bar{\varepsilon}_{33}^{S}$ (Eq. 15 ). From these coefficients, it is possible to determine the effective parameters $\left(\bar{Z}_{L}, \bar{c}_{L}\right.$ and $\left.\bar{k}_{t}\right)$ of the piezocomposite. From the relation $(14)$, we can write:

$$
T_{3}=\bar{c}_{33}^{D} S_{3}-\bar{h}_{33} D_{3}
$$

Then, it can be deduced:

$$
\left\{\begin{array}{l}
\bar{c}_{33}^{D}=\bar{c}_{33}^{E}+\frac{\left(\bar{e}_{33}\right)^{2}}{\bar{\varepsilon}_{33}^{S}} \\
\bar{h}_{33}=\frac{\bar{e}_{33}}{\bar{\varepsilon}_{33}^{S}}
\end{array}\right.
$$

Therefore, from the effective mass density $\bar{\rho}=v_{f} \rho^{f}+v_{m} \rho^{m}$ we can express the various effective parameters as bellow:

$$
\left\{\begin{array}{l}
\bar{Z}_{L}=\sqrt{\bar{c}_{33}^{D} \bar{\rho}} \\
\bar{c}_{L}=\sqrt{\frac{\bar{c}_{33}^{D}}{\bar{\rho}^{D}}} \\
\bar{k}_{t}=\frac{\bar{e}_{33}}{\sqrt{\bar{c}_{33}^{D} \bar{\varepsilon}_{33}^{S}}}
\end{array}\right.
$$

\section{Results}

To estimate the numerical values of the effective coefficients and parameters of piezocomposite 1-3, we call on the developments of section 2. In this study, the piezoelectric materials PVDF-TrFE copolymer (polyvinylidene fluoride-trifluoroethylene) and PZT-5A are used as phase (inclusion) and phase 2 (matrix), respectively. The coefficients of the piezocomposite used in the study are presented in Table 1 . The main purpose of the study is to assess the influence of the volume fraction and the active properties of the matrix on the effective electromechanical properties of 1-3 piezocomposite. To compare our results with
Table 1: Electromechanical properties of piezoelectric materials 33

\begin{tabular}{ccccccccc}
\hline Materials & $\begin{array}{c}c_{11}^{E} \\
(\mathrm{GPa})\end{array}$ & $\begin{array}{c}c_{12}^{E} \\
(\mathrm{GPa})\end{array}$ & $\begin{array}{c}c_{13}^{E} \\
(\mathrm{GPa})\end{array}$ & $\begin{array}{c}c_{33}^{E} \\
(\mathrm{GPa})\end{array}$ & $\begin{array}{c}e_{31} \\
\left(\mathrm{c} . \mathrm{m}^{-2}\right)\end{array}$ & $\begin{array}{c}e_{33} \\
\left(\mathrm{c} . \mathrm{m}^{-2}\right)\end{array}$ & $\begin{array}{c}\rho \\
\left(\mathrm{kg} . \mathrm{m}^{-3}\right)\end{array}$ & $\frac{\varepsilon_{33}^{s}}{\varepsilon_{0}}$ \\
\hline PVDF-TrFE & 8.5 & 3.6 & 3.6 & 9.9 & 0.008 & -0.29 & 1880 & 7.5 \\
PZT-5A & 126 & 79.5 & 84.1 & 109 & -6.5 & 24.8 & 7898 & 1813 \\
\hline
\end{tabular}

the literature, numerical data has been extracted from [33] and experimental data from [28].

In this work, some electromechanical coefficients and effective parameters of 1-3 piezocomposite have been established via an extended analytical method based on the Smith model, which takes into account only one piezoelectic phase. As demonstrated in the previous section, the proposed model takes into account two piezoelectric phases. Those resulting effective coefficients of the piezocomposite are studied, then compared and discussed through the influence of the volume fraction $v_{f}$ of the PZT-5A piezoelectric phase. This model developed in view to evaluate the piezocomposite behavior while in service, made it appear that the homogenized coefficients are model dependent but differ little.

For values of the volume fraction between 0 and 0.8 , a rather slow evolution can be noticed for the elastic coefficient $\bar{c}_{33}^{E}$ (Figure 2 (a)). This remark is observed for the present models as well as for other models or the Reuss terminal which is the lower limit. We also note that the matrix model coincides with the Smith model graphically. For the volume fraction values between 0.8 and 1 , an exponential evolution of this coefficient is observed.

The effective piezoelectric coefficient $\bar{e}_{33}$ (Figure 2 (b)) has linear evolution for the two models with a volume fraction between 0 and 0.9 whereas a parabolic evolution is observed for a volume fraction between 0.9 and 1 . For the dielectric coefficient $\bar{\varepsilon}_{33}^{S} / \varepsilon_{0}$ (Figure $2(\mathrm{c})$ ), the evolution of two representative curves of these models evolves linearly with the volume fraction; as is the Reusss model. With respect to the effective electromechanical coupling coefficient $\bar{k}_{t}$ (Figure 2 (d)), the effective longitudinal wave velocity $\bar{c}_{L}$ (Figure $2(\mathrm{e})$ ) and the effective acoustical impedance $\bar{Z}_{L}$ (Figure 2 (f)) of the piezocomposite, the Reuss model constitutes the lower bound.

Results that were expected because of the impedance formulation that is a function of the elastic characteristics. It should be noted, however, that for the electromechanical coupling coefficient for values of the volume fraction between 0.2 and 0.8 the Reuss bound is slightly above this homogenization technique. As for the longitudinal velocity, we realize that the variations are important because of the difference between the three curves. These different variations of the longitudinal velocity must not be neglected because it is important for determining the resonant frequency of the piezoelectric transducer made from piezoelectric materials. Since the performance of a transducer is related to the electromechanical coupling coefficient of the piezo-composite, this parameter deserves a thorough study. The Reuss model has achieved a value greater than $70 \%$. In general, the values of this parameter for 1-3 piezo-composite are above $50 \%$ for volume fractions between $10 \%$ to more than $90 \%$. 


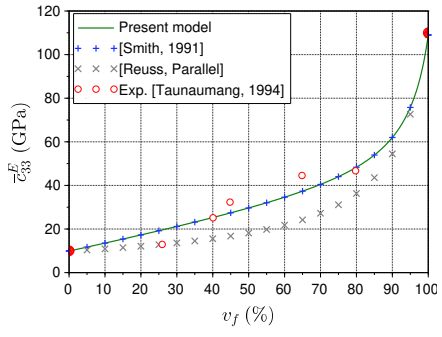

(a)

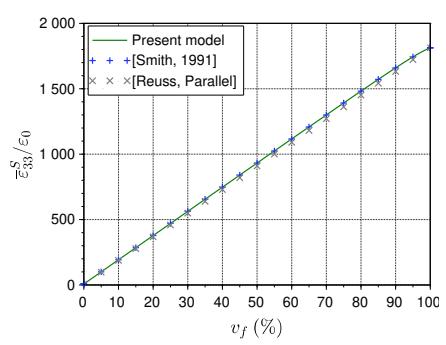

(c)

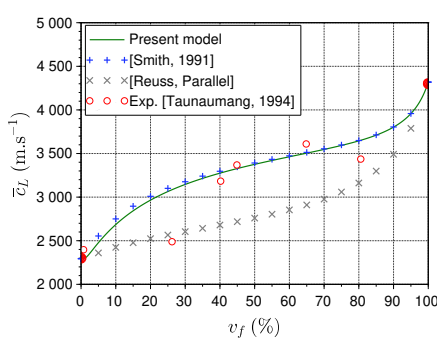

(e)

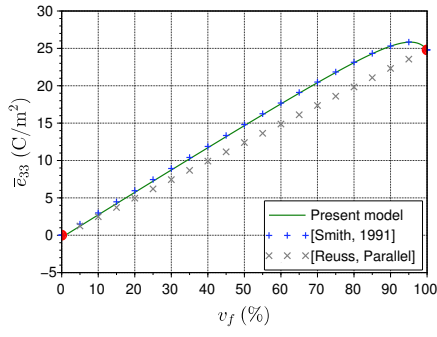

(b)

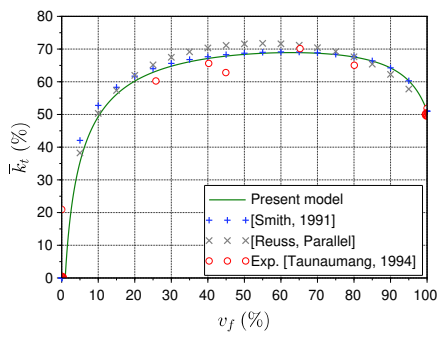

(d)

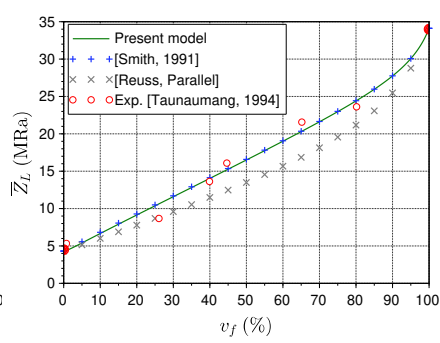

(f)
Figure 2: Representation of homogenized coefficients and actual parameters of 1-3 piezocomposite made of a PZT-5A / PVDF-TrFE as a function of the volume fraction $v_{f}$ : (a) elastic coefficient at constant electric field $\bar{c}_{33}^{E}$, (b) piezoelectric coefficient $\bar{e}_{33}$, (c) relative dielectric coefficient at constant strain $\bar{\varepsilon}_{33} / \varepsilon_{0}$, (d) thickness coupling coefficient $\bar{k}_{t}$, (e) longitudinal wave velocity $\bar{c}_{L}$ and (f) longitudinal acoustical impedance $\bar{Z}_{L}$.

This explains the fact that 1-3 piezocomposite is the most used in manufacturing of transducers. Our results are in agreement with those obtained experimentally by Taunaumang [28]. In short, we consider that here the elastic, piezoelectric and dielectric coefficients on the one hand and on the other hand the effective characteristics present two zones of study. The evolution of these coefficients can be subdivided into two zones: a relatively slow growth zone according to the volume fraction (between 0 and 0.8 ) and a fast growth zone (zone between 0.8 and 1).

\section{Underwater applications}

Piezocomposite with two active phases can be used in the manufacture of new ultrasonic transducers. In these transducers, ceramic is used for signal emission and the copolymer provides a better electrical and acoustic correspondence to the input and output signals [33]. The fact that the two phases are active implies the opposition of the sign of piezoelectric coefficients and this makes it possible to place these phases in opposite directions therefore improve the piezoelectric coupling effect that is used in the design of the robust transducers. In the literature [28, 33, 34], there are few works where the two phases are piezoelectrically active. Therefore, the study of the effective properties of these piezocomposites is of paramount importance.

Such piezocomposites are typically used either for ultrasonic underwater transducers or hydrophone applications [28, 33, 34]. Their performance can estimated on the basis of specific coefficients, taking into account the hydrostatic environment. Among those figures of merit (FOM), one can cite the hydrostatic charge $\bar{d}_{h}$, the hydrostatic voltage coefficient $\bar{g}_{h}$, the hydrophone trade-off $\bar{d}_{h} \bar{g}_{h}$ or the hydrostatic electromechanical coupling factor $\bar{k}_{h}$. These are defined as follows:

$$
\left\{\begin{array}{l}
\bar{d}_{h}=\bar{d}_{31}+\bar{d}_{32}+\bar{d}_{33} \\
\bar{s}_{h}=2 \bar{s}_{11}+4 \bar{s}_{13}+\bar{s}_{33} \\
\bar{g}_{h}=\frac{\bar{d}_{h}}{\sqrt{\bar{s}_{h} \bar{\varepsilon}_{33}^{T}}}
\end{array}\right.
$$

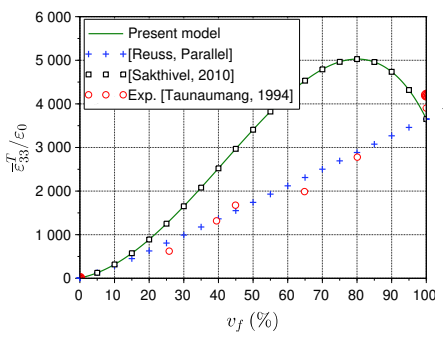

(a)

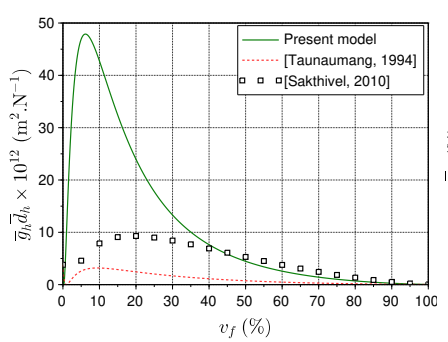

(c)

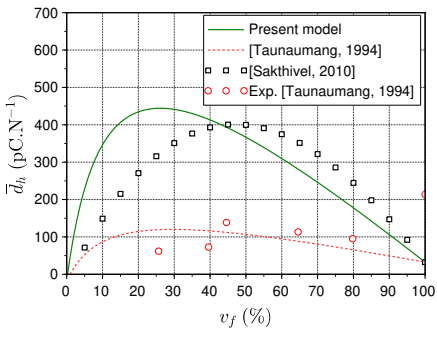

(b)

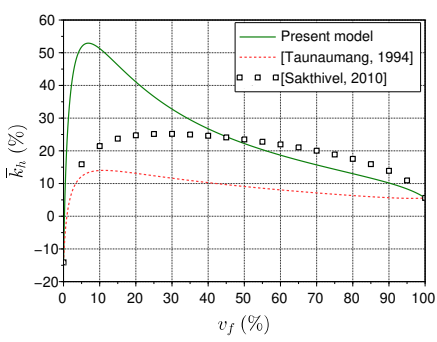

(d)
Figure 3: Homogenized electroacoustic properties of the PZT-5A / PVDF-TrFE 1-3 piezocomposite as a function of the PZT-5A volume fraction $v_{f}$ : present model, Reuss model [15] and experimental data from Taunaumang et al. [28]: (a) relative dielectric coefficient at constant stress $\bar{\varepsilon}_{33}^{T} / \varepsilon_{0}$, (b) effective hydrostatic charge constant $\bar{d}_{h}$, (c) hydrostatic trade-off $\bar{g}_{h} \bar{d}_{h}$ (d) hydrostatic electromechanical coupling factor constant $\bar{k}_{h}$

In the studied PZT-5A / PVDF-TrFE 1-3 piezocomposite composition (Table 1), the homogenized piezoelectric properties are analyzed though indirect coefficients deduced from the homogenized $\left\{\bar{c}_{i j}^{E}, \bar{e}_{i j}, \bar{\varepsilon}_{i j}^{S}\right\}$ piezoelectric matrix data. As a result, the relative dielectric coefficient at constant $\bar{\varepsilon}_{33}^{T} / \varepsilon_{0}$ Figure 3 (a)) shows a positive hybrid effect with a maximum value reaching 5000 for $v_{f}=80 \%$. Concerning the hydrostatic charge $\bar{d}_{h}$ 
(Figure 3 (b)), the maximum value reaches 450 pC.N ${ }^{-1}$, contrary to Taunaumang [28] both numerically and experimentally. The mismatch with the curve obtained with the Sakthivel model [33] seems to be due to the values of dij taken into account for the plot, which are differing from those obtained with the calculation from the $\left\{c_{i j}^{E}, e_{i j}, \varepsilon_{i j}^{S}\right\}$ initial set of values. Due to this, the values of the hydrophone trade-off $\bar{k}_{h} \bar{g}_{h}$ (Figure 3 (c)) obtained with the proposed model are drastically different, but the maximum value is very near to that of Taunaumang [28], but quite different from the model of Sakthivel [33], mainly resulting from the differing $\bar{d}_{h}$ coefficient. As a combination of those FOM, the hydrostatic coupling factor $\bar{k}_{h}$ (Figure $3(\mathrm{~d})$ ) is different from that of Taunaumang [28], itself quite different from the Sakthivel model [33]. Those values are significantly lower than those of the thickness coupling coefficient $\bar{k}_{t}$ (Figure $2(\mathrm{~d})$ ). This is explained by the fact that the hydrostatic coupling factor $\bar{k}_{h}$ evaluates the overall acoustic to electric power conversion, whereas the thickness coupling coefficient $\bar{k}_{t}$ is only focusing on the thickness mode efficiency. Nevertheless, those FOM do not take into account the electrical and acoustical environments properly. In that view, a specific transducer application field is to be chosen, defined and analyzed as a design for the targeted application.

\section{Transducers for imaging application}

Once the properties the piezocomposite material are defined, its properties have to be taken into account in the end-user application. Commonly, the electroacoustic response of transducers is calculated using an electroacoustic equivalent model, the KLM model for instance [35]. In this view, the surrounding layers and media have to be simulated. In a first approach, the electric matching with the generator and the acoustic matching with the back and front media are of first importance. The acoustic impedance of the rear face of a piezoelectric transducer has a damping effect on the pseudo-harmonic resonance of the piezoelectric layer alone. Its value is to be chosen as a trade-off between sensitivity and bandwidth, for example using a performance index PI designed depending on the targeted application [32, 35, 36]. Thus, the studied configuration deals with a medical imaging application, i.e. a piezoelectric transducer radiating in water. Some additional specific characteristics are fixed such as pulse-echo generator having a low electric impedance $Z_{g}=Z_{g}=5 \Omega$, a PZT-5A piezoelectric material having a thickness resonance mode at $f_{0}=25 \mathrm{MHz}$, with an active diameter of 0.5 " corresponding to an active surface of $S=40 \mathrm{~mm}^{2}$, a front medium having an acoustic impedance $Z_{f}=1.5 \mathrm{MRa}$. As a starting point, the effect of the damping is first considered on the PZT-5A in view to determine the bandwidth and sensitivity evolution. Then, in a seond step, the piezocomposite composition of PZT-5A / PVDF-TrFE with the optimal value of the PZT-5A volume fraction. Thus, some simulations are carried out using the KLM model, varying the acoustic impedance of the backing through the backing load ratio $Z_{b} / Z_{p}$. As a re- sult, the pulse-echo transfer function is evaluated with the impulse response spectrum $\operatorname{IRS}(f)$ (Figure 4 (a)) and the associated electroacoustic echo $E A E(t)=F T^{-1}\{\operatorname{IRS}(f)\}$ (Figure 4 (b)). These electroacoustic responses are compared through the amplitude, center frequency and relative bandwidth are in Figure 4 (c). In addition, two estimators are built from their characteristics and plotted in Figure 4 (d). Thus, the bandwidth flatness $B W F$ is defined as follows:

$$
B W F=\frac{1}{B W_{6}} \int_{f_{6}, \min }^{f_{6}, \max } \frac{|\operatorname{IRS}(f)|}{\max \{|I R S(f)|\}} d f
$$

Moreover, the product of the relative bandwidth by the relative amplitude defines the $B W A$ estimator:

$$
B W A=B W_{6, r} a m p_{r}
$$
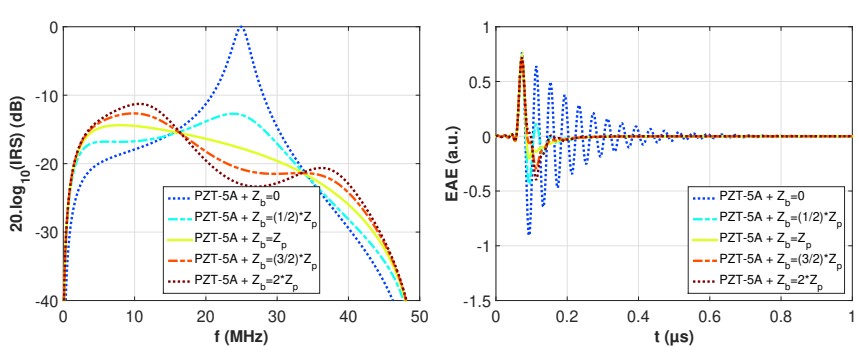

(a)

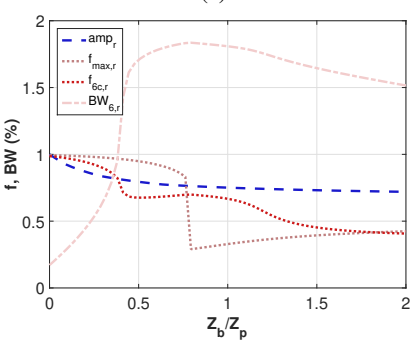

(c) (b)

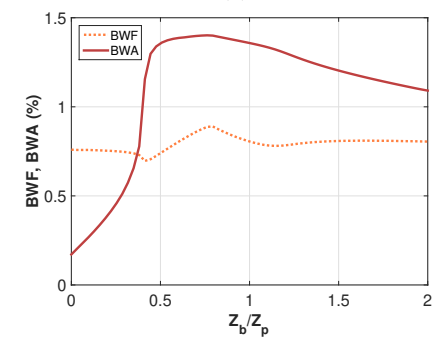

(d)
Figure 4: Comparison of PZT-5A based piezocomposites transducers radiating in water with a loaded backing from $Z_{b}=0$ to $2 . Z_{p}$ in terms of (a) impulse response spectrum $I R S$, (b) electroacoustic echo $E A E$; their associated characteristics: (c) normalized amplitude $a m p_{r}$, center frequencies $f_{6 c, r}$ and $f_{\text {max }, r}$, and relative bandwidth $B W_{6, r}$ and (d) bandwidth based estimators: the bandwidth flatness $B W F$ as defined in Eq. 20) and the normalized bandwidth by amplitude product $B W A$ as defined in Eq. 21,

On the basis of the simulation results, the impulse response spectrum $\operatorname{IRS}(f)$ is illustrated in Figure 4 (a) for the backing load ratio $Z_{b} / Z_{p}$, from 0 to 2 , with a step of 0.5 . It clearly shows two phenomena: first the damping of the resonance between the case $Z_{b} / Z_{p}=0$ (no backing) and $Z_{b} / Z_{p}=0.5$ (very light backing); second, the frequency corresponding to the maximum peak of the spectrum $f_{\text {max, } r}$ is shown to decrease nearly by a factor 2 between the case $Z_{b} / Z_{p}=0.5$ (very light backing) and $Z_{b} / Z_{p}=1.5$ (heavy backing). This second phenomenon can be observed more clearly in Figure 4 (c) with the the plot of the $f_{\text {max }, r}$ extracted parameter. In Figure $4(\mathrm{~b})$, the appearance of the associated electroacoustic echo $E A E(t)=F T^{-1}\{I R S(f)\}$ can be observed to evolve from a poorly damped resonance when $Z_{b} / Z_{p}=0$ (no backing, but damping from the front load) 
to the damped resonance when $Z_{b} / Z_{p}=0.5$ (very light backing). For higher values of the backing load ratio $Z_{b} / Z_{p}$, the electroacoustic echo $E A E(t)$ exhibits some distorsions, and the echo duration do not decrease anymore. This echo duration is known to be inversely proportional to the bandwidth, itself related to the image resolution. However, a tradeoff is to be found with the sensitivity which is strongly affected in the same time. As illustrated by Figure 4 (c), the relative amplitude $a m p_{r}$ decreases regularly as a function the of the backing load ratio $Z_{b} / Z_{p}$, whereas the center frequencies $f_{\text {max }, r}$ and $f_{6 c, r}$ are showing some transitions while decreasing. More particularly, the frequency corresponding to the maximum peak of the spectrum $f_{\text {max }, r}$ exhibits a sudden transition between $Z_{b} / Z_{p}=0.75$ and $Z_{b} / Z_{p}=0.80$. The center frequency $f_{6 c, r}$ shows a first transition around $Z_{b} / Z_{p}=0.4$, and a second smooth one around 1.2. This center frequency $f_{6 c, r}$ smooth estimator thus constitutes a reliable parameter for an optimization procedure. On the basis of these curves illustrated in Figure 4 (d), the backing load ratio $Z_{b} / Z_{p}$ giving the maximum bandwidth flatness $B W F$ (Eq. 20p) is observed to coincide with that of the normalized bandwidth by amplitude product $B W A$ (Eq. 21). This maximum is obtained for $Z_{b} / Z_{p}=0.82$, and is therefore considered as an optimum value for the studied configuration. As illustrated in Figure $4(\mathrm{~d})$, the variation of the estimator $B W A$ shows a maximum, whereas the estimator $B W F$ varies more slowly and less clearly. Moreover, this latter estimator has the same shape as that of the relative bandwidth $B W_{6, r}$ (Figure 4 (c)) which is that commonly used for ultrasound imaging applications. Indeed, this $B W A$ estimator takes the amplitude into account which is also an important parameter for the image quality, through the signal-to-noise ratio.

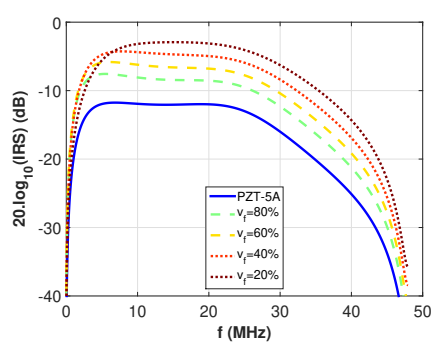

(a)

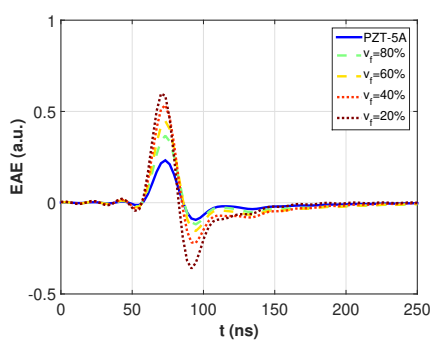

(b)
Figure 5: Performance of immersion piezocomposite transducers equiped with a loaded epoxy backing where $Z_{b} / Z_{p}=0.82$. Influence of the PZT-5A volume fraction $v_{f}=100,80,60,40,20 \%$ on the homogenized 1-3 piezocomposite made of a PZT-5A / PVDF-TrFE composition: (a) impulse response spectrum $I R S$, (b) electroacoustic echo EAE.

Depending on the targeted application, the sensitivity of volume fraction $v_{f}$ is determined from the data obtained in Section 3 (Figure 2) and illustrated in Figure 5. As a result, the optimal piezocomposite composition exhibit a higher sensitivity but the relative bandwidth and resonance frequency may be lower, so that an optimization procedure is to be defined for the determination of the optimum value of the normalized backing impedance $Z_{b} / Z_{p}$. Thus, in the simulated configuration, the relative bandwidth shows a maximum value for $v_{f}=80 \%$ where $B W_{6, r}=184,187,185,180$ and $168 \%$ while the normalized amplitude increases with $a m p_{r}=0.76,1.19,1.46,1.77$ and 2.08. These values are resulting in a normalized bandwidth by amplitude product $B W A=1.4,2.2,2.7,3.2$ and 3.5, for a volume fraction $v_{f}=100,80,60,40,20 \%$ respectively. These results can be explained by the acoustic matching between the front face of the transducer $Z_{L}$ (Figure 2 (f)) and the propagation medium $Z_{f}=1.5 \mathrm{MRa}$. Nevertheless, for values $v_{f}<20 \%$, the electroacoustic response characteristics are drastically devalued due to the lower values of the thickness coupling coefficient $k_{t}$ (Figure 2 (d)).

\section{Conclusion}

In this work, it was demonstrated that a specific combination of piezoelectric phases can lead to new optimal values for piezocomposite transducers. An analytical method based on the Smith model has been generalized, leading to deduce the actual parameters in terms of elastic, piezoelectric and dielectric coefficients. The optimization of piezocomposite composition such as the PZT-5A / PVDF-TrFE composition was shown to be not only a question of effective electromechanical parameters, but also a question of the accurate study of the end-user specifications. The transducer performance is often discussed in terms of a bandwidth-sensitivity trade-off, what is developed and argued with additional estimators such as the bandwidth flatness $B W F$ and the bandwidth by amplitude product $B W A$. The possibilities offered by those piezocomposite compositions are very sensitive to the considered piezoelectric phases and their volume fraction. As a result, these simulations are aimed at studying effect of the volume fraction of a constituent (piezoelectric ceramic phase) on the homogenized piezocomposite coefficients in order to predict its behavior while in service. In the future work, we intend to study the association of two connectivities in order to deduce also these same properties and make a comparison.

\section{Data Availability}

The processed data required to reproduce these findings cannot be shared at this time as the data also forms part of an ongoing study.

\section{References}

[1] R. C. Smith, Smart Material Systems: Model Development, Society for Industrial and Applied Mathematics, 2005. doi:10.1137/1. 9780898717471

[2] V. Y. Topolov, C. R. Bowen, Effective electromechanical properties in piezocomposites (2009) 11-41doi : 10.1007/978-1-84882-000-5_2

[3] W. Smith, B. Auld, Modeling 1-3 composite piezoelectrics: thicknessmode oscillations, IEEE Transactions on Ultrasonics, Ferroelectrics, and Frequency Control 38 (1) (1991) 40-47, conference Name: IEEE Transactions on Ultrasonics, Ferroelectrics, and Frequency Control. doi: $10.1109 / 58.67833$

[4] A. Agbossou, C. Richard, Y. Vigier, Segmented piezoelectric fiber composite for vibration control: fabricating and modeling of electromechanical properties, Composites Science and Technology 63 (6) (2003) 871881. doi:10.1016/S0266-3538(02)00298-1

[5] L. J. Nelson, Smart piezoelectric Fibre composites, Materials Science and Technology 18 (11) (2002) 1245-1256. doi:10.1179/ 026708302225007448 
[6] K. Uchino, Ferroelectric devices, Boca Raton: CRC press, 2009. doi: $10.1201 / \mathrm{b} 15852$

[7] K. A. Klicker, J. V. Biggers, R. E. Newnham, Composites of PZT and Epoxy for Hydrostatic Transducer Applications, Journal of the American Ceramic Society 64 (1) (1981) 5-9. doi:10.1111/j.1151-2916. 1981.tb09549.x

[8] M. Avellaneda, P. J. Swart, Calculating the performance of 1-3 piezoelectric composites for hydrophone applications: An effective medium approach, The Journal of the Acoustical Society of America 103 (3) (1998) 1449-1467. doi:10.1121/1.421306

[9] C. P. Chong, W. P. Chen, H. L. W. Chan, P. C. K. Liu, Nonlinear behavior of piezoceramics and piezocomposites under various ac fields, Sensors and Actuators A: Physical 116 (2) (2004) 320-328. doi:10.1016/j. sna.2004.04.013

[10] H. Liu, J. Zhong, C. Lee, S.-W. Lee, L. Lin, A comprehensive review on piezoelectric energy harvesting technology: Materials, mechanisms, and applications, Applied Physics Reviews 5 (4) (2018) 041306. doi : 10.1063/1.5074184

[11] Z. Yang, S. Zhou, J. Zu, D. Inman, High-performance piezoelectric energy harvesters and their applications, Joule 2 (4) (2018) 642-697. doi:10.1016/j.joule.2018.03.011

[12] G.-M. Yang, O. Obi, G. Wen, N. X. Sun, Design of tunable bandpass filters with ferrite sandwich materials by using a piezoelectric transducer, IEEE Transactions on Magnetics 47 (10) (2011) 3732-3735, conference Name: IEEE Transactions on Magnetics. doi:10.1109/TMAG.2011. 2155037

[13] P. Benech, J. M. Duchamp, Piezoelectric materials and their applications in radio frequency domain and telecommunications, Advances in Applied Ceramics 114 (4) (2015) 220-225. doi:10.1179/1743676115Y. 0000000009

[14] H. J. Lee, S. Zhang, Y. Bar-Cohen, S. Sherrit, High temperature, high power piezoelectric composite transducers, Sensors 14 (8) (2014) 1452614552. doi:10.3390/s140814526

[15] R. E. Newnham, D. P. Skinner, L. E. Cross, Connectivity and piezoelectric-pyroelectric composites, Materials Research Bulletin 13 (5) (1978) 525-536. doi:10.1016/0025-5408(78)90161-7

[16] H. Banno, Recent developments of piezoelectric ceramic products and composites of synthetic rubber and piezoelectric ceramic particles, Ferroelectrics 50 (1) (1983) 3-12. doi:10.1080/00150198308014425

[17] T. Furukawa, K. Fujino, E. Fukada, Electromechanical Properties in the Composites of Epoxy Resin and PZT Ceramics, Japanese Journal of Applied Physics 15 (11) (1976) 2119. doi:10.1143/JJAP.15.2119

[18] R. Steinhausen, T. Hauke, W. Seifert, H. Beige, W. Watzka, S. Seifert, D. Sporn, S. Starke, A. Schönecker, Finescaled piezoelectric 1-3 composites: properties and modeling, Journal of the European Ceramic Society 19 (6) (1999) 1289-1293. doi:10.1016/S0955-2219(98)00422-1

[19] H. L. W. Chan, P. K. L. Ng, C. L. Choy, Effect of poling procedure on the properties of lead zirconate titanate/vinylidene fluoride-trifluoroethylene composites, Applied Physics Letters 74 (20) (1999) 3029-3031. doi : 10.1063/1.124054

[20] K. L. Ng, H. L. W. Chan, C. L. Choy, Piezoelectric and pyroelectric properties of pzt/p(VDF-TrFE) composites with constituent phases poled in parallel or antiparallel directions, IEEE Transactions on Ultrasonics, Ferroelectrics, and Frequency Control 47 (6) (2000) 1308-1315. doi:10.1109/58.883519

[21] M. L. Dunn, M. Taya, Micromechanics predictions of the effective electroelastic moduli of piezoelectric composites, International Journal of Solids and Structures 30 (2) (1993) 161-175. doi:10.1016/ 0020-7683(93) 90058-F

[22] W.-S. Kuo, J. H. Huang, On the effective electroelastic properties of piezoelectric composites containing spatially oriented inclusions, International Journal of Solids and Structures 34 (19) (1997) 2445-2461. doi:10.1016/S0020-7683(96)00154-0

[23] C.-W. Nan, L. Liu, D. Guo, L. Li, Calculations of the effective properties of 1-3 type piezoelectric composites with various rod/fibre orientations, Journal of Physics D: Applied Physics 33 (22) (2000) 2977-2984. doi : 10.1088/0022-3727/33/22/317

[24] G. M. Odegard, Constitutive modeling of piezoelectric polymer composites, Acta Materialia 52 (18) (2004) 5315-5330. doi:10.1016/j . actamat.2004.07.037

[25] R. Kar-Gupta, T. A. Venkatesh, Electromechanical response of 1-3 piezo- electric composites: An analytical model, Acta Materialia 55 (3) (2007) 1093-1108. doi:10.1016/j.actamat.2006.09.023

[26] K. Hashimoto, M. Yarnaguchi, Elastic, piezoelectric and dielectric properties of composite materials, IEEE International Ultrasonics Symposium (IUS) (1986) 697-702 doi:10.1063/1.357099

[27] W. Smith, Limits to the enhancement of piezoelectric transducers achievable by materials engineering, IEEE International Ultrasonics Symposium (IUS) (1992) 697-702 doi : 10.1109/ULTSYM.1992 . 275912

[28] H. Taunaumang, I. L. Guy, H. L. W. Chan, Electromechanical properties of 1-3 piezoelectric ceramic/piezoelectric polymer composites, Journal of Applied Physics 76 (1) (1994) 484-489. doi:10.1063/1.357099

[29] C. N. Della, D. Shu, The performance of 1-3 piezoelectric composites with a porous non-piezoelectric matrix, Acta Materialia 56 (4) (2008) 754-761. doi:10.1016/j.actamat.2007.10.022

[30] F. Levassort, M. Lethiecq, D. Certon, F. Patat, A matrix method for modeling electroelastic moduli of 0-3 piezo-composites, IEEE Transactions on Ultrasonics, Ferroelectrics, and Frequency Control 44 (2) (1997) 445452. doi:10.1109/58.585129

[31] A. Bale, F. Levassort, A.-C. Hladky-Hennion, Coupled model for determination of full electroelastic sets of 1-3 piezocomposite with various pillar shapes, in: 2017 IEEE International Ultrasonics Symposium (IUS), 2017, pp. 1-4, iSSN: 1948-5727. doi : 10.1109/ULTSYM. 2017. 8091841

[32] F. L. Hanse Wampo, R. P. Lemanle Sanga, P. Maréchal, G. E. Ntamack, Piezocomposite transducer design and performance for high resolution ultrasound imaging transducers, International Journal of Computational Materials Science and Engineering 08 (03) (2019) 1950013. doi:10. 1142/S2047684119500131

[33] M. Sakthivel, A. Arockiarajan, An analytical model for predicting thermo-electro-mechanical response of 1-3 piezoelectric composites, Computational Materials Science 48 (4) (2010) 759-767. doi : 10.1016/ j.commatsci.2010.03.027

[34] L. V. Gibiansky, S. Torquato, On the use of homogenization theory to design optimal piezocomposites for hydrophone applications, Journal of the Mechanics and Physics of Solids 45 (5) (1997) 689-708. doi:10. 1016/S0022-5096(96)00106-8

[35] P. Maréchal, F. Levassort, L.-P. Tran-Huu-Hue, M. Lethiecq, Lensfocused transducer modeling using an extended KLM model, Ultrasonics 46 (2) (2007) 155-167. doi:10.1016/j.ultras .2007.01.006

[36] P. Marechal, F. Levassort, Electroacoustic response at the focal point of a focused transducer as a function of the acoustical properties of the lens Proceedings of 5th World Congress on Ultrasonics (2003) 535-538. URL http://www.conforg.fr/wcu2003/procs/cd1/articles/ 000248.pdf 